\title{
Outcome of Pregnancy with Intrauterine Device In Situ: A Meta-analysis
}

\author{
Eka R Gunardi ${ }^{1}$, Raymond Surya ${ }^{2}$
}

\begin{abstract}
Introduction: Intrauterine devices (IUDs) constitute the second-most used contraceptive method worldwide. Pregnancy in the presence of IUD results in obstetric complications. This study aims to review the outcome of intrauterine pregnancies among IUD removed, IUD retained, and no IUD.

Materials and methods: There were 89, 8, and 52 studies found in Pubmed ${ }^{\oplus}$, Cochrane Library ${ }^{\oplus}$, and Ovid ${ }^{\circledR}$ database. We included cohort or case-control studies consisting of minimally two groups: (i) no IUD and IUD retained or (ii) IUD removed and retained. Several outcomes were assessed, including preterm birth, miscarriage, premature rupture of membrane (PROM), placental abruption, placental previa, intrauterine growth restriction, chorioamnionitis, and cesarean delivery. The analysis of this meta-analysis used review manager 5.3.

Results: There were 7 studies included owing to language barrier and accessibility of article. Pregnancy with IUD in situ increased the risk of miscarriage (RR 6.50; 95\% Cl 4.56-9.28), PROM (RR 1.88; 95\% Cl 0.98-3.62), placenta previa (RR 2.33; 95\% Cl 1.14-4.73), placental abruption (RR 4.51; 95\% Cl 2.82-7.20), chorioamnionitis (RR 6.07; 95\% Cl 3.91-9.42), and cesarean delivery (RR 1.33; 95\% Cl 1.03-1.71). Meanwhile, IUD removed decreased the risk of miscarriage ( $\mathrm{RR} 0.51 ; 95 \% \mathrm{Cl} 0.39-0.66$ ) and preterm birth ( $\mathrm{RR} 0.57,95 \% \mathrm{Cl} 0.38-0.86$ ) compared with IUD retained. Conclusion: Conceiving with IUD in pregnancy increases the rate of miscarriage, placenta previa, abruption, chorioamnionitis, and cesarean delivery. Meanwhile, IUD removed early in pregnancy decreases the rate of miscarriage and preterm birth.

Keywords: Intrauterine device, Intrauterine pregnancy, Outcome.

Journal of South Asian Federation of Obstetrics and Gynaecology (2019): 10.5005/jp-journals-10006-1689
\end{abstract}

\section{INTRODUCTION}

Intrauterine devices (IUDs) constitute the second-most used contraceptive method worldwide; it is safe, effective, and widely used as the fifth most common among the modern contraception methods in USA.' In Indonesia, on the basis of Profil Kesehatan Indonesia 2016, IUD users became the fourth highest contraceptivedevice users after injection, pill, and implant users, with a prevalence of $7.23 \%$ and $10.61 \%$ among new and active users, respectively. ${ }^{2}$ The failure rate of this method ranges from $0.8 \%$ to $2.3 \%{ }^{3,4}$ The presence of IUD causes local inflammation through releasing prostaglandins and leukocytes from the endometrium (also progesterone from certain device) to create a hostile environment for oocytes and sperms. Besides, copper has a spermatocytic effect to prevent pregnancy. ${ }^{5}$

Pregnancy in the presence of IUD results in obstetric complications; therefore, removal of IUD during the first trimester of pregnancy is recommended to prevent septic complication and miscarriages. ${ }^{6,7}$ Hadas et al. ${ }^{8}$ stated that IUD retained in pregnancy increased several risks such as preterm delivery and chorioamnionitis. Meanwhile, on the basis of studies from 1970s to 1980 s with a limited number of samples, the World Health Organization (WHO) recommends ruling out of ectopic pregnancy in the presence of IUD. Apart from that, removing IUD improves pregnancy outcomes if the IUD strings are visible or can be retrieved safely from the cervical canal. ${ }^{9}$ However, there are still limited studies discussing between IUD removed and retained in pregnancy to the outcome. Therefore, this study aims to review the outcome of intrauterine pregnancy among IUD removed, IUD retained, and no IUD.
1,2Department of Obstetrics and Gynecology, Dr Cipto Mangunkusumo Hospital, Faculty of Medicine, Universitas Indonesia, Jakarta, Indonesia Corresponding Author: Raymond Surya, Department of Obstetrics and Gynecology, Dr Cipto Mangunkusumo Hospital, Faculty of Medicine, Universitas Indonesia, Jakarta, Indonesia, Phone: +62 81320000288, e-mail: raymond_s130291@yahoo.co.id

How to cite this article: Gunardi ER, Surya R. Outcome of Pregnancy with Intrauterine Device In Situ: A Meta-analysis. J South Asian Feder Obst Gynae 2019;11(3):212-216.

Source of support: Nil

Conflict of interest: None

\section{Materials and Methods}

\section{Criteria for Considering Studies for this Review}

Types of Studies

Cohort and case-control studies that investigate the intrauterine pregnancy outcome either IUD retained vs no IUD or IUD removed vs retained.

\section{Type of Participants}

Studies that described intrauterine pregnancy outcomes in women who had Copper T 380A-IUD in situ during conception.

\section{Type of Comparison}

The studies included in our review should consist of minimally two groups: no IUD and IUD retained, or IUD removed and retained.

() The Author(s). 2019 Open Access This article is distributed under the terms of the Creative Commons Attribution 4.0 International License (https://creativecommons. org/licenses/by-nc/4.0/), which permits unrestricted use, distribution, and non-commercial reproduction in any medium, provided you give appropriate credit to the original author(s) and the source, provide a link to the Creative Commons license, and indicate if changes were made. The Creative Commons Public Domain Dedication waiver (http://creativecommons.org/publicdomain/zero/1.0/) applies to the data made available in this article, unless otherwise stated. 


\section{Type of Outcome}

We assessed several outcomes, including preterm birth, miscarriage, premature rupture of membrane (PROM), placental abruption, placental previa, intrauterine growth restriction, chorioamnionitis, and cesarean delivery.

\section{Search Methods for Identification of Studies}

We did not impose any language or other restrictions on the beginning of searches.

\section{Electronic Searches}

The search was conducted on Pubmed ${ }^{\oplus}$, Cochrane Library ${ }^{\oplus}$, and Ovid $^{\circledast}$. In PubMed, the search included keywords using the $\mathrm{MeSH}$, namely "Pregnancy outcome AND Intrauterine Devices". Meanwhile, in Cochrane, the MeSH descriptor consisted of (Pregnancy) AND (Intrauterine device). The author used keywords of intrauterine pregnancy and (pregnancy complication or pregnancy outcome) and an intrauterine device in Ovid. In this searching strategy (performed on August 15th 2018), there were 89, 8, and 52 studies in Pubmed ${ }^{\oplus}$, Cochrane Library ${ }^{\oplus}$, and Ovid ${ }^{\oplus}$ database, respectively. The articles were screened using criteria such as abstracts answering the clinical questions, written in English language, full-text paper availability, and omitting all duplication papers. The analysis of this meta-analysis used review manager 5.3.

\section{Data Collection and Analysis}

\section{Selection of Studies}

Our search generated a list of abstracts. Two review authors (RS and ER) independently screened these abstracts. Studies that were not relevant were excluded at this stage. Any uncertainty on the eligibility of the studies that was based on title and abstract made the reviewers read full paper. The study flow diagram was shown in Flowchart 1.

\section{Assessment of Risk of Bias in Included Studies}

The risk of bias within the study was assessed using the Cochrane risk of bias table.

\section{Assessment of Heterogeneity}

Heterogeneity was assessed through the score of $R^{2}$, which consisted of $P^{2}<50 \%$ as homogeneity among studies.

\section{RESULTS}

The best study design to answer prognostic question is cohort studies. In this review, there were 10 articles related to the topic; however, only 7 studies included owing to language barrier and accessibility of article. The flow of literature through the assessment process for the update of this review is shown in Flowchart 1.

\section{Included Studies}

The studies included in meta-analysis are shown in Table 1.

\section{Risk of Bias included Studies}

Figure 1 described the risk of bias summary. Allocation concealment, blinding of participants and personnel, and blinding of outcome assessment could not be determined owing to prognostic study.

\section{Pregnancy Outcomes}

Of the studies included in the meta-analysis, there were several pregnancy outcomes between IUD and no IUD (Table 2) and IUD removed and retained (Table 3).

\section{Discussion}

The limitation of this review was no proceedings of conferences were included and timing of IUD removal was not specified clearly. Of the studies included in this meta-analysis, there were three studies showing unclear or high risk of selection bias, such as Howard et al., ${ }^{10}$ Skjeldestad et al., ${ }^{7}$ and Chaim et al. ${ }^{11}$ The first study by Howard et al. ${ }^{10}$ did not mention clearly whether subjects were asked for the time of IUD removal. Meanwhile, Skjeldestad et al. ${ }^{7}$ started the study from all pregnant women to search the presence of IUD and Chaim et al. ${ }^{11}$ designed a case-control study by comparing 1 and 3 for case and control subjects. However, this was the first meta-analysis which summarized all studies focusing on IUD removed, retained, and no IUD to the pregnancy outcome.

Flowchart 1: Study flow diagram in this review

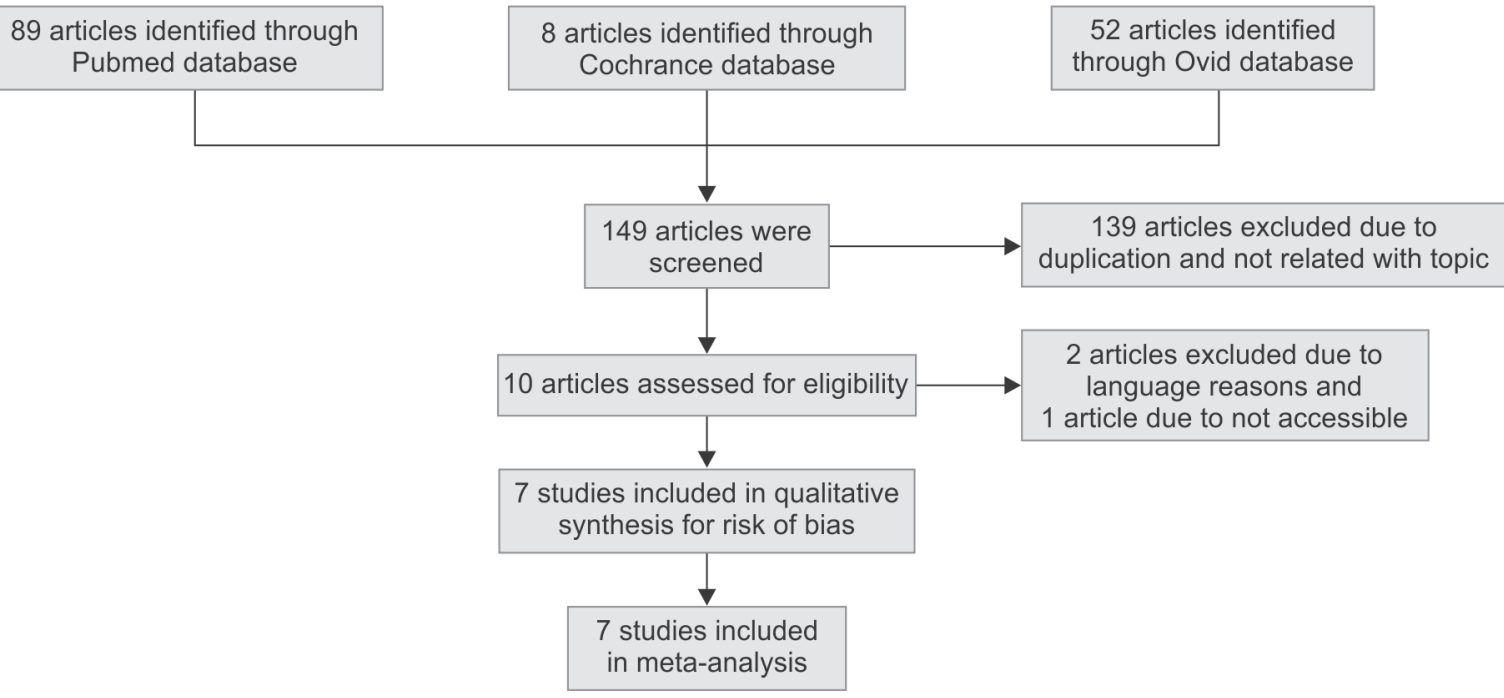




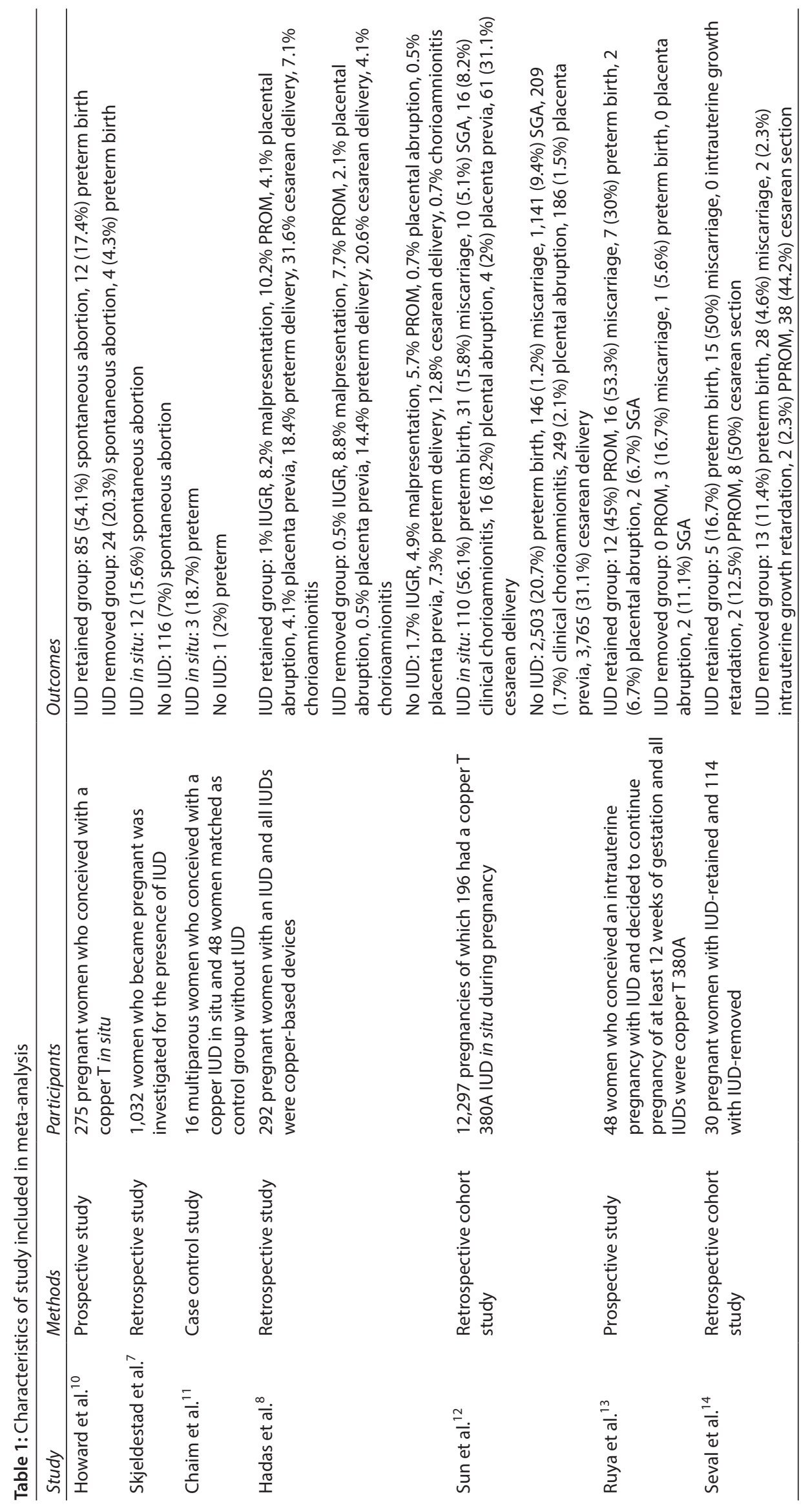


Conceiving with IUD and Pregnancy Outcome

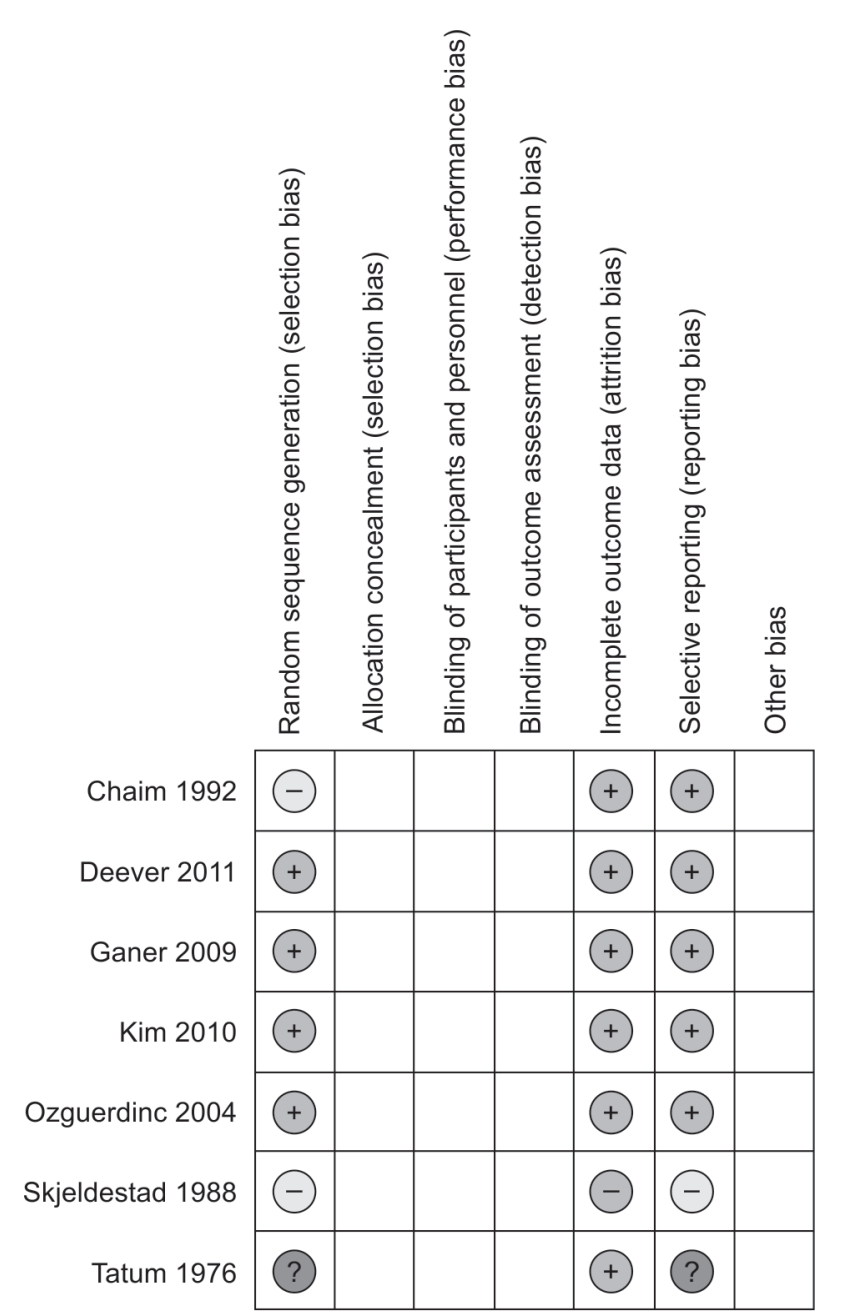

Fig. 1: Risk of bias summary for each study

Table 2: Outcomes of pregnancy in group of IUD and no IUD

\begin{tabular}{lllll}
\hline IUD vs No IUD & $R R$ & $95 \% \mathrm{Cl}$ & $p$ & $l^{2}$ \\
\hline Miscarriage & 6.50 & $4.56-9.28$ & $<0.001$ & $96 \%$ \\
Preterm birth & 1.06 & $0.91-1.24$ & 0.47 & $91 \%$ \\
PROM & 1.88 & $0.98-3.62$ & 0.06 & $\mathrm{~N} / \mathrm{A}$ \\
Placenta previa & 2.33 & $1.14-4.73$ & 0.02 & $87 \%$ \\
Placental abruption & 4.51 & $2.82-7.20$ & $<0.001$ & $0 \%$ \\
Chorioamnionitis & 6.07 & $3.91-9.42$ & $<0.01$ & $63 \%$ \\
Intrauterine growth & 0.95 & $0.59-1.50$ & 0.81 & $97 \%$ \\
restriction & & & & \\
Cesarean delivery & 1.33 & $1.03-1.71$ & 0.03 & $93 \%$ \\
\hline
\end{tabular}

$\mathrm{RR}$, relative risk; $\mathrm{Cl}$, confidence interval

PROM, premature rupture of membrane; N/A, not available

Of all studies that included IUD retained and removed, only studies by Seval et al. ${ }^{14}$ and Ruya et al. ${ }^{13}$ used ultrasound examination to reveal visible copper IUD in the uterine cavity. After this examination, Ruya et al. ${ }^{13}$ and Hadas et al. ${ }^{8}$ showed explicitly that IUD removal was performed in the early gestation, namely first 12 weeks of gestation (trimester 1). Meanwhile, other studies did not mention clearly the time of IUD removal.

Compared with pregnancy without IUD, women who conceived with IUD had a higher rate of placental abruption. A previous
Table 3: Outcomes of pregnancy in group of IUD removed and retained

\begin{tabular}{lllll}
\hline IUD removed vs retained & $R R$ & $95 \% \mathrm{Cl}$ & $p$ & $l^{2}$ \\
\hline Miscarriage & 0.51 & $0.39-0.66$ & $<0.001$ & $85 \%$ \\
Preterm birth & 0.57 & $0.38-0.86$ & 0.007 & $33 \%$ \\
PROM & 0.51 & $0.25-1.05$ & 0.07 & $62 \%$ \\
Placenta previa & 0.13 & $0.01-1.11$ & 0.06 & $\mathrm{~N} / \mathrm{A}$ \\
Placental abruption & 0.51 & $0.13-1.98$ & 0.33 & $\mathrm{~N} / \mathrm{A}$ \\
Chorioamnionitis & 0.58 & $0.22-1.55$ & 0.27 & $\mathrm{~N} / \mathrm{A}$ \\
Intrauterine growth & 0.82 & $0.11-5.93$ & 0.84 & $0 \%$ \\
restriction & & & & \\
Cesarean delivery & 0.79 & $0.57-1.08$ & 0.14 & $29 \%$ \\
\hline
\end{tabular}

$\mathrm{RR}$, relative risk; $\mathrm{Cl}$, confidence interval

PROM, premature rupture of membrane; N/A, not available

study stated that chronic inflammatory process of placenta lead to placental abruption. Apart from that, chronic inflammatory process activated cytokines, such as interleukin-1 $\beta$ and tumor necrosis factor- $a{ }^{15}$ This was also associated with chorioamnionitis. Neutrophil infiltration to fetal membrane ended to preterm PROM and placental abruption, suggesting that enhanced protease activity and inflammatory cytokines had a role in placental abruption. ${ }^{16}$ Sun et al. ${ }^{12}$ suggested that inflammatory state due to IUD in situ during pregnancy contributed to the risk of placental abruption, preterm PROM, spontaneous preterm labor with intact membrane. In this meta-analysis, the author found all complications related to IUD in pregnancy caused a higher rate of cesarean delivery.

This meta-analysis revealed that conceiving with IUD leads to significant risk factors for maternal outcomes. The IUD removed early in pregnancy decreased the rate of miscarriage and preterm birth; however, an IUD in pregnancy increased the risk of miscarriage, placenta previa, placental abruption, chorioamnionitis, and cesarean delivery compared with normal pregnancy without IUD. It was corresponding to previous systematic review in 2012, which showed that women with a retained IUD had a greater risk for spontaneous abortion, preterm delivery, and septic abortion. Meanwhile, compared with women who conceived without IUD, this systematic review showed a higher risk for spontaneous abortion, preterm delivery, and chorioamnionitis even after the IUD removal in early pregnancy. ${ }^{17}$ Chorioamnionitis in IUD retained is reactive inflammation owing to the presence of a foreign body; so it evolve to secondary infection; ${ }^{8}$ however, IUD removal in this metaanalysis was not differently significant to IUD retained in pregnancy.

\section{ConCLUSION}

Conceiving with IUD in pregnancy increases the rate of miscarriage, placenta previa, abruption, chorioamnionitis, and cesarean delivery. Meanwhile, IUD removed early in pregnancy decreases the rate of miscarriage and preterm birth.

\section{References}

1. Mosher WD, Jones J. Use of contraception in the United States: 19822008. Vital Health Stat 232010 Aug;(29):1-44.

2. Indonesia KKR. Data dan Informasi Profil Kesehatan Indonesia 2016. Pus Data Dan Inf Kementrian Keshatan RI; 2017. pp. 119-121.

3. de Araujo FF, Barbieri M, Guazzelli CAF, et al. The T 380A intrauterine device: a retrospective 5-year evaluation. Contraception 2008 Dec;78(6):474-478. DOI: 10.1016/j.contraception.2008.07.006.

4. WHO|Selected practice recommendations for contraceptive use [Internet]. WHO. [cited 2018 Aug 15]. Available from: http://www.who. int/reproductivehealth/publications/family_planning/SPR-3/en/. 
5. Stanford JB, Mikolajczyk RT. Mechanisms of action of intrauterine devices: update and estimation of postfertilization effects. Am J Obstet Gynecol 2002 Dec;187(6):1699-1708. DOI: 10.1067/mob.2002.128091.

6. Horn LC, Nenoff P, Ziegert M, et al. Missed abortion complicated by Candida infection in a woman with rested IUD. Arch Gynecol Obstet 2001 Jan;264(4):215-217. DOI: 10.1007/s004040000117.

7. Skjeldestad FE, Hammervold R, Peterson DR. Outcomes of pregnancy with an IUD in situ-a population based case-control study. Adv Contracept Off J Soc Adv Contracept 1988 Dec;4(4):265-270. DOI: 10.1007/BF01849267.

8. Ganer H, Levy A, Ohel I, et al. Pregnancy outcome in women with an intrauterine contraceptive device. Am J Obstet Gynecol 2009 Oct;201(4):381.e1-381.e5. DOI: 10.1016/j.ajog.2009.06.031.

9. Hoopes AJ, Simmons KB, Godfrey EM, et al. 2016 Updates to US Medical Eligibility Criteria for Contraceptive Use and Selected Practice Recommendations for Contraceptive Use: Highlights for Adolescent Patients. J Pediatr Adolesc Gynecol 2017;30(2):149-155. DOI: 10.1016/ j.jpag.2017.01.013.

10. Tatum HJ, Schmidt FH, Jain AK. Management and outcome of pregnancies associated with the Copper T intrauterine contraceptive device. Am J Obstet Gynecol 1976 Dec 1;126(7):869-879. DOI: 10.1016/0002-9378(76)90675-X.

11. Chaim W, Mazor M. Pregnancy with an intrauterine device in situ and preterm delivery. Arch Gynecol Obstet 1992;252(1):21-24. DOI: 10.1007/BF02389602.
12. Kim SK, Romero R, Kusanovic JP, et al. The prognosis of pregnancy conceived despite the presence of an intrauterine device (IUD). J Perinat Med 2010;38(1):45-53. DOI: 10.1515/jpm. 2009.133.

13. Deveer R, Engin-Ustun Y, Sarikaya E, et al. Comparison of C-reactive protein levels in pregnancies with retained and removed intrauterine device. J Matern-Fetal Neonatal Med Off J Eur Assoc Perinat Med Fed Asia Ocean Perinat Soc Int Soc Perinat Obstet 2011 Sep;24(9): 1152-1154. DOI: 10.3109/14767058.2010.545925.

14. Ozgu-Erdinc AS, Tasdemir UG, Uygur D, et al. Outcome of intrauterine pregnancies with intrauterine device in place and effects of device location on prognosis. Contraception 2014 May;89(5):426-430. DOI: 10.1016/j.contraception.2014.01.002.

15. Ananth CV, Oyelese $\mathrm{Y}$, Prasad V, et al. Evidence of placental abruption as a chronic process: associations with vaginal bleeding early in pregnancy and placental lesions. Eur J Obstet Gynecol Reprod Biol 2006 Oct;128(1-2):15-21. DOI: 10.1016/j.ejogrb.2006. 01.016.

16. Nath CA, Ananth CV, Smulian JC, et al. New Jersey-Placental Abruption Study Investigators. Histologic evidence of inflammation and risk of placental abruption. Am J Obstet Gynecol 2007 Sep;197(3):319.e1-319. e6. DOI: 10.1016/j.ajog.2007.06.012.

17. Brahmi D, Steenland MW, Renner R-M, et al. Pregnancy outcomes with an IUD in situ: a systematic review. Contraception $2012 \mathrm{Feb}$;85(2): 131-139. DOI: 10.1016/j.contraception.2011.06.010. 